\title{
A Therapentic Approach for Two Patients with Multiple Supernumerary and Impacted Teeth
}

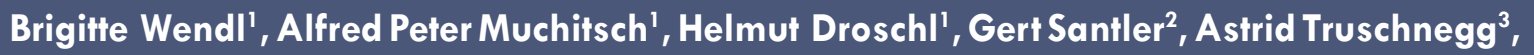 \\ Norbert Jakse ${ }^{3}$ \\ 'Department of Orthodontics and Dentofacial Orthopedics, ${ }^{3}$ Department of Oral Surgery and \\ Radiology, Medical University of Graz, Austria, Auenbruggerplatz 12, A-8036 Graz, Austria; \\ ${ }^{2}$ Department of Oral Surgery and Radiology - Medical University of Wels-Grieskirchen, 4710 \\ Grieskirchen, Austria.
}

Abstract:

The occurrence of multiple supernumerary teeth in the absence of systemic disease is uncommon and therapy requires interdisciplinary collaboration. We hereby report cases and treatment process of two brothers having 10 and 7 supernumerary and impacted teeth with class III symptoms.

Key words: Cysts, Dentition, Impacted Tooth, Supernumerary Tooth, Tooth Eruption.

\section{Introduction}

The aetiology of supernumerary teeth has not yet been fully clarified. Different theories exist, ranging from tooth bud divisions, through hyperactivity of the dental lamina to hereditary disposition [1]. Prevalence is stated to be $0.8 \%$ for deciduous teeth and $2.1 \%$ for permanent teeth [2], whereby the manifestation can vary (simple, multiple, uni-bilateral, non-erupted or erupted supernumerary teeth) [3]. $76 \%-86 \%$ of cases are classed as simple, whereas $12 \%-23 \%$ involve duplicated and less than $1 \%$ multiple supernumerary teeth [4]. Most surplus teeth tend to be located in the anterior upper jaw area [5], followed by lower jaw pre-molars and molars.

Whilst for deciduous teeth there are no gender-specific incidence differences, the permanent teeth of males are more often affected with a
2:1 ratio $[6,7]$. The occurrence of supernumerary teeth is very often combined with other medical conditions or syndromes (Cleidocranial dysplasia, Gardners syndrome, cleft lip and/or palate) [5]. The occurrence of multiple supernumerary teeth is uncommon in the absence of systemic or syndromal disease. Only 6 cases with a family history of supernumerary teeth without systemic symptoms or syndromes are described in the literature.

Four different types can be distinguished on the basis of shape, namely: conical, bumpy, supplementary-duplicated and ontodoma [8], whereby the conical form with an incidence of $70 \%$ is the most frequent variant [5]. A study by Esenlik et al. [9] showed that $74 \%$ of diagnosed supernumerary teeth are impacted. The authors

Corresponding Author: Priv. Doz. Dr. Brigitte Wendl

Email: brigitte.wendl@medunigraz.at

Received: October 24, 2015 | Accepted: December 31, 2015 | Published Online: February 5, 2016

This is an Open Access article distributed under the terms of the Creative Commons Attribution License (creativecommons.org/licenses/by/3.0)

Conflict of interest: None declared | Source of funding: Nil | DOl: http://dx.doi.org/10.17659/01.2016.0014 
attribute delayed eruption and later impaction to a reduced rate of root growth and subsequent reduced spacing. The possible consequences of supernumerary teeth range from cyst formation, displacements, crowding and delayed eruption to impaction and permanent tooth root resorption [8]. The present paper describes two treatment cases - two brothers with supernumerary and impacted teeth and the interdisciplinary collaboration of the treatment.

\section{Case Reports:}

Assessment of Orthodontic Diagnosis and Findings

Case 1 is a 16 year old male patient with mandibular pro and maxillary retrognathia as well as bilateral cross-bite. Dental status showed inverted overbite in the front teeth area and a class III occlusion with a $3 / 4$ pre-molar width [Fig.1]. The panoramic X-ray and computer tomograph revealed a duplication of both maxillary incisors, an additional rudimentary tooth bud 15, a tooth 19, a supernumerary anterior mandibular tooth, a triplication of both mandibular 2nd pre-molars as well as a duplication of tooth 34 [Fig.2]. All maxillary anterior teeth, both canines and teeth 34 and 35 were impacted (in total, 10 impacted teeth). The CT results otherwise showed unremarkable jaw articulation and no bone destruction or dissolution.

Case 2, the younger brother of case 1 (11 years), also exhibited a class-IIl-syndrome with mandibular pro and maxillary retrognathia and cross bite in the molar areas. Dental status also showed inverted overbite and a class III occlusion of a $1 / 2$ pre-molar width. The upper jaw exhibited a duplication of both central incisors, of tooth 14 as well as a mesiodent. Tooth 21 was impacted. The mandibular $2^{\text {nd }}$ pre-molars were duplicated and triplicated on the right and left sides of the jaw respectively [Fig.3].

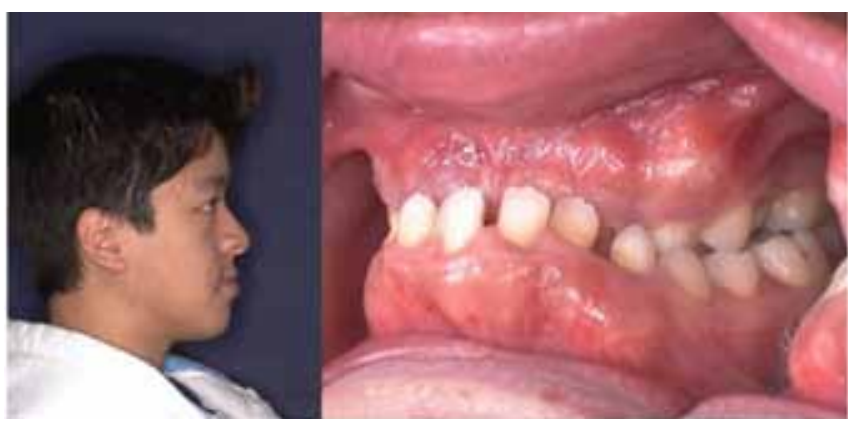

Fig.1: Profile photo showing mandibular pro- and maxillary retrognathia. Mouth-internal photo of a deeply reversed overbite in the front. Dental status showed inverted overbite in the front teeth area and a class III occlusion with a $3 / 4$ pre-molar width [Fig.1].

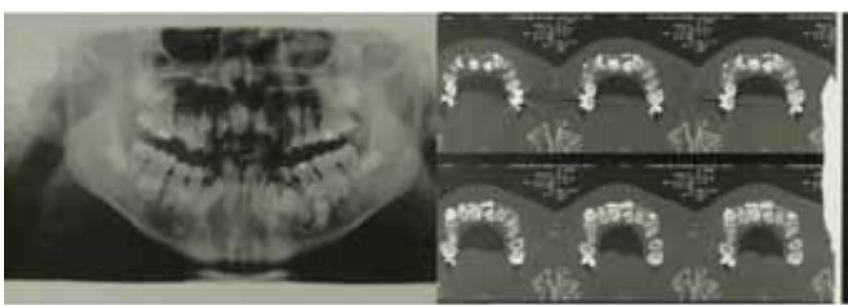

Fig.2: Panorama X-ray with multiple supernumerary and impacted teeth. The CTimage shows multiple supernumerary and impacted teeth.

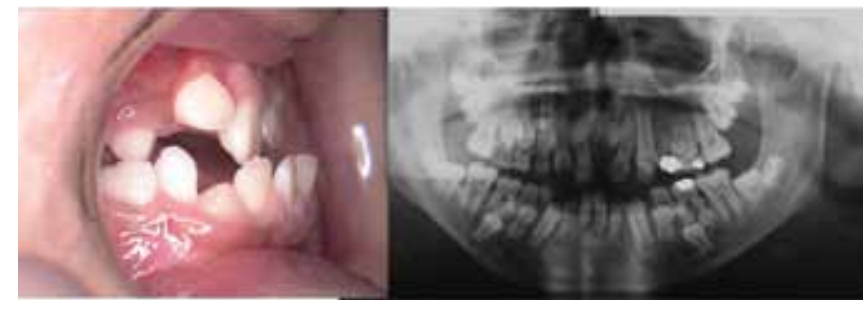

Fig.3: Mouth-internal photo with a deeply reversed overbite in the front. Panorama X-ray with multiple supernumerary and impacted teeth. 


\section{Treatment Objectives}

In Case 1 first the surgical removal of the supernumerary teeth and the deciduous teeth under general anaesthesia was planned, followed by the operative exposure and bonding of the impacted teeth and subsequently alignment. The correction of the bilateral cross bite should be managed by conventional expansion. The correction of the sagittaly discrepancy resp. inverted overbite was planned by surgical intervention.

In Case 2 the first step was the surgical removal of the supernumerary teeth and the deciduous teeth under general anaesthesia, followed by the operative exposure and bonding of the impacted teeth 21 and subsequently alignment. The correction of the cross bite was planned by conventional expansion. The correction of the sagittaly discrepancy resp. inverted overbite should be done surgically after completion of the growth.

\section{Treatment Alternatives}

The transverse discrepancy concerning case 1 could also be corrected by a Le Fort I osteotomy in two parts. But this procedure is combined with higher surgical complexity and relapse. During surgical removal of the supernumerary teeth under general anaesthesia, some of the impacted teeth could have been removed and later on replaced by dental implants. But the orthodontic treatment with the possibility for alignment was necessary anyway, so this approach was not an option.

The correction of the sagittal discrepancy of patient 1 had to be done surgically because of the amount of discrepancy and age. Concerning patient 2, a maxillary protraction with a bone-anchored miniplate in the maxilla and mandible and class III elastics, like Hugo De Clerk shows, would have been possible. Also a Hybrid Hyrax Mentoplate Combination [10] would have been an option.
These possibilities were refused by the patient. A face mask therapy could not be used because of the dental situation (eruption and alignment of the premolars, canines and the impacted front teeth after surgical removal of the supernumerary teeth).

\section{Treatment Progress}

Case 1: The bilateral cross bite exhibited by the older of the two patients was corrected by transversal expansion using a Haas device (with soldered hooks to fix the elastics for the alignment of the impacted teeth) [Fig.4]. After removal of the palatal-placed duplication of teeth 11,21 , the rudimentary tooth bud 15 and the deciduous teeth from 53-63, teeth $13,12,11,21,22$ and 23 were exposed and bonded to gold chains. This surgical procedure was carried out under general anaesthesia.

Alignment of the retained, bonded teeth [Fig.5] was planned with elastics und tension

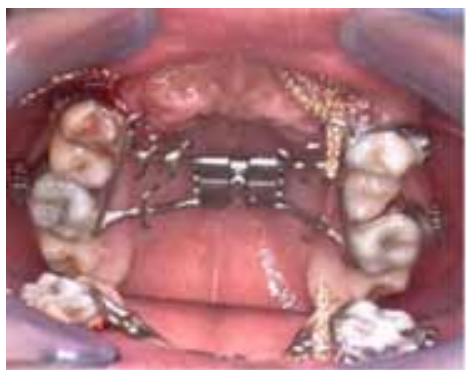

Fig.4: Haas device (with soldered hooks).

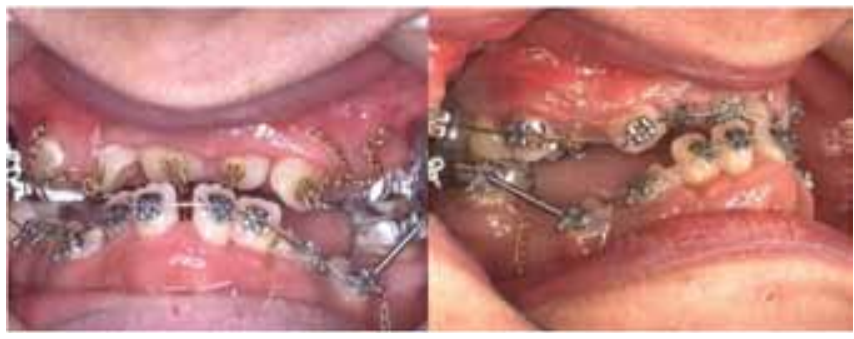

Fig.5: Tooth Exposure of and bonding of a gold chain to the teeth. Regulation of the impacted, bonded teeth using elastics and tension springs. 
springs. After the extrusion of the central maxillary incisors with tension springs against the Haas device the lateral incisors were to be subsequently regulated, also with support against the Haas device. Moderate forces of $60-90 \mathrm{cN}$ were to be applied in the process, because of the higher risk of root resorption for the anterior maxillary teeth. The maxillary and mandibular canines were to be symmetrically extruded with elastic rubber cords.

The leftmost mandibular front tooth was extracted due to an existing dilaceration. The remaining deciduous teeth and the lingual located supernumerary mandibular pre-molar tooth bud right and left were moreover extracted. During the same sitting, the permanent impacted canines and pre-molars 34 and 45 were bonded with gold chains in order to subsequently bring them in place through closed eruption. The permanent first molars, and thereby the necessary space in the lateral support zone, were maintained by means of a mandibular lip bumper. A Le Fort I osteotomy under general anaesthesia was planned following the successful alignment of all permanent teeth and the creation of two harmonious dental arches. This combined orthodontic-oral surgical combination therapy was unavoidable due to the age of the patient and the severity of the skeletal mal-positioning.

Case 2: The younger patient's cross bite was corrected using a trans-palatal bracket with pre-molar arms and hooks. During the same sitting tooth 21 was exposed and bonded to a small chain. Here too alignment was planned through closed eruption with the force directed against the hook on the trans-palatal bracket. The supernumerary teeth were surgically removed and all deciduous teeth extracted except for $65,75,85$. A maxillary part-bonding with frontal teeth brackets $11,53,22$ and light pressure coil springs 11-22 was then carried out. After space opening for tooth 21, the latter was extruded in the planned tension direction with an elastic thread. An overlay mechanic was subsequently used. A lip bumper was employed in the lower jaw to hold the first molars in place until the eruption of all permanent teeth [Fig.6]. After bonding of all teeth, the transversal distance was still supported with the trans-palatal bracket and the spaces held open with commensurate distance tubes [Fig.7].

Results

Case 1: After post-operative fine adjustments, a class I occlusion and harmonic profile were achieved for the older patient [Fig.8].

Case 2: Because the younger patient was still growing, a retention aligner was inserted after the creation of two harmonious tooth arches. This holding phase will be maintained until the planned orthognathic operation [Fig.9].

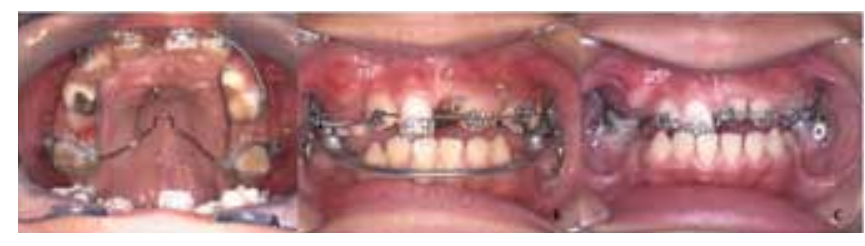

Fig.6: (A) Trans palatal-bracket with premolar arms and hooks. (B) Extrusion of tooth 21 with an elastic cord, a Lip bumper in the lower jaw to hold the first molars in position. (C) Overlay mechanic.

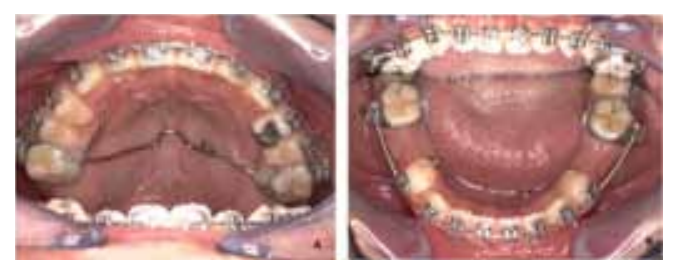

Fig.7: (A) Trans-palatal bracket to support the transversal width. (B) Gap maintenance with commensurate distance tubes. 
In both cases the periodontal conditions were good with the attached gingiva almost normally configured or only minimally shortened. The final panorama X-ray revealed minor root resorption and dilacerations of the aligned (previously impacted) teeth [Fig.10]. The prognosis for these can however be classified as very favourable.

\section{Discussion}

The incidence of multiple supernumerary deciduous teeth exhibits no gender dependency. The permanent teeth of males however, (our cases) are affected twice as often as those of females [6]. The patients presented in this case report are descended from a Chinese mother. According to literature reports [8], persons from Asian countries are more frequently affected than other peoples.

Whilst mesiodents occur very often as supernumerary teeth, their incidence in the anterior mandible is in contrast only $0.01 \%$ [11]. In our cases even a supernumerary mandibular frontal tooth was present. The indications for extraction of supernumerary teeth or for further monitoring without extraction should follow specific criteria (delayed eruption of permanent teeth, resorptions, displacement of teeth, the ensuing orthodontic treatment) [5]. Di Biase [12] found that $75 \%$ of incisors erupted spontaneously following the extraction of supernumerary teeth. Given sufficient space, eruption took, on average, 18 months. Witsenburg and Boering [13] described a markedly lower eruption frequency (54\%) and recommended that the remaining tooth be routinely bonded to a chain during the surgical procedure. The successful alignment of impacted teeth is further dependent on the following factors: (i) position und alignment of the impacted tooth, (ii) progress of root formation, (iii) dilaceration grade, (iv) adequate space for the impacted tooth that is to be aligned [14]. In the cases described here, orthodontic treatment was definitely necessary

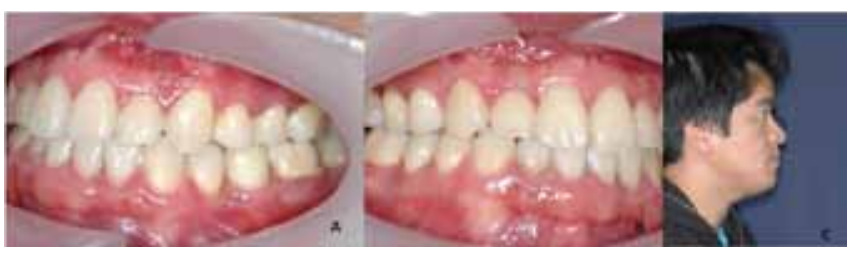

Fig.8: (A,B) Mouth-internal photo showing a class I occlusion. (C) Profile photo (patient 1) following orthognathic surgery.

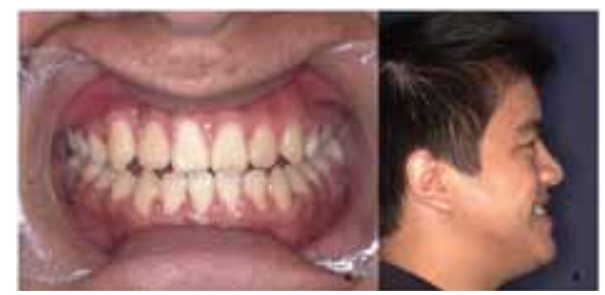

Fig.9: (A) Mouth-internal photo following regulation of the impacted teeth. (B) Preoperative profile photo (patient 2) following the regulation of the impacted teeth.

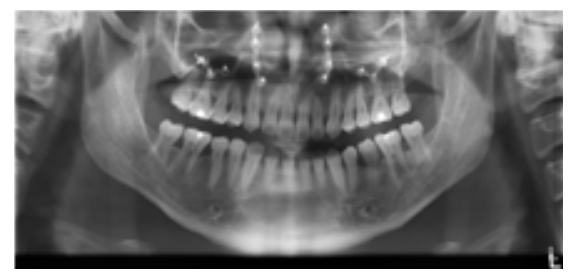

Fig.10: Panorama X-ray following orthognathic surgery.

due to the class III symptomatic and the application of proportionate traction was necessary due to already existing root formation. Following the extraction of the supernumerary teeth, the impacted, permanent teeth were therefore bonded with a gold chain during the same sitting for postoperative orthodontic alignment.

A good radiological and clinical diagnosis (Panorama X-ray and computer tomography were carried out) are decisive in the design of the 
treatment plan. A digital volume tomography (DVT, Cone Beam CT) would have been also an alternative. These radiological methods were not available at that time of treatment planning. Raupp $S$ et al. [15] describe the successful use of CT diagnosis even for a five year old girl. The planning and execution of the surgical procedure, as well as the operation time and post-operative process can be significantly improved by precise diagnostics. Exact radiological diagnosis is a must for cases of supernumerary teeth. The impacted area can cause considerable problems, in particular when, as in the present cases, both supernumerary and impacted teeth are present. The positioning of the teeth in relation to the neighbouring root is of great importance, both for the decision regarding the extraction of supernumerary teeth and for their regulation following surgical exposure. The movement vector along which the extrusion and subsequent regulation is to occur should be pre-operatively defined. Based on the presence of multiple impacted teeth, patient comfort considerations, the more favourable long term periodontal result and due to the orthodontic treatment that is to immediately follow we selected a closed eruption [16]. But also the conditions of the gingiva and the position of the impacted teeth (mainly buccal) were reasons to choose this eruption technique [17].

For the surgical removal of the supernumerary teeth, especially in the mandibular pre-molar region, certain stages are given preference complete tooth development, complete crowns with on-going root development and complete crown formation [18]. In the present case, the extraction decision was governed by the stage of root growth and the possibility for alignment. Tooth buds and teeth with non-age appropriate root development were first and foremost removed. The work of Esenlik et al. showed that supernumerary premolars root development is less than that of the normal neighbouring teeth. All of these had a supplementary form.
Interdisciplinary collaboration is unavoidable for a successful outcome of complex orthodontic treatments. In our case, intensive surgical planning discussions, extraction decisions, removal of the wisdom teeth and tooth 19, an appropriate pre-operative set-up and the preparation of the Splint for the Le Fort I osteotomy were necessary. Concerning patient 2 a maxillary protraction with a bone-anchored miniplate in the maxilla and mandible and class III elastics would have been possible. De Clerk [19] describes in his work an average increment on skeletal and soft-tissue advancement of maxillary structures of about $4 \mathrm{~mm}$ and favourable mandibular changes of $2 \mathrm{~mm}$.

Overall, a treatment intervention due to supernumerary teeth can be considered to belong to the known interceptive orthodontic early treatments [20], in particular because the prognosis for the active placement of impacted teeth is more favourable prior to complete root development. Although the medical histories contained no description of a familial occurrence of supernumerary tooth, a genetic disposition appears to be very likely in this case (siblings). Batra et al. [21] postulated autosomal dominant transmission in their work. Whilst the abnormal occurrence of supernumerary teeth does not follow simple Mendelian inheritance rules [3], the class III syndrome most definitely does however. Whether a correlation exists between class III syndromes and supernumerary teeth, has not be determined by the literature.

\section{Conclusion}

In the present examples, interdisciplinary collaboration enabled the achievement of normal occlusion, the avoidance of long term damage (cyst formation, impaction and root resorption of permanent teeth) and the permanent (Case 1) and temporary discharging (Case 2 - treatment pause) respectively of self-confident young gentlemen. 
In cases of delayed tooth eruption and impacted permanent teeth, thought should definitely be given to the presence of supernumerary teeth and appropriate diagnostic and therapeutic treatment steps considered.

\section{References}

1. Garvey MT, Barry HJ, Blake M. Supernumerary Teeth - An Overview of Classification, Diagnosis and Management. J Can Dent Assoc. 1999;65:612-616.

2. Brook AH. Dental anomalies of number, form and size: their pre valence in British school children. J Int Assoc Dent Child. 1974;5(2):37-53.

3. Scheiner MA, Sampson WJ. Supernumerary teeth: a review of the literature and four case reports. Aust Dent J. 1997;42:160-165.

4. Orhan Al, Özer L. Familial Occurrence of Nonsyndromal multiple Supernumerary teeth. Angle Orthodontist. 2006;76:891-897.

5. Schmuckli R, Lipowsky C, Peltomäki T. Prevalence and Morphology of Supernumerary Teeth in the population of a Swiss Community. Schweiz Monatsschr Zahnmed. 2010;1 20:987-990.

6. Kinirons MJ. Unerupted premaxillary supernumerary teeth. A study of their occurrence in males and females. Br Dent J. 1982;153:1 10.

7. Mitchel L. Supernumerary teeth. Dent Update. 1989; 16:65-69.

8. Singh S, Prerna, Gupta P. Multiple Supernumerary Teeth - A Case Report. Indian Journal of Dental Sciences. 2009;1:1-4.

9. Esenlik E, Sayin MÖ, Atilla OA, Özen T, Altun C, Basak F. Supernumerary teeth in a Turkish population. Am J Orthod Dentofac Orthop. 2009; 136:848-852.

10. Wilmes $B$, Nienkemper $M$, Ludwig $B$, Kau CH, Drescher D. Early Class III treatment with a hybrid hyrax-mentoplate combination. J Clin Orthod. $2011 ; 45(1): 15-21$; quiz 39.

11. Yokose T, Sakamoto T, Sueishi K, Yatabe $\mathrm{K}$, Tsujiino K, Kubo S, et al. Two cases with supernumerary teeth in Lower Incisor Region. Bull Tokyo Dent Coll. 2006;47(1):19-23.

12. Di Biase DD. The effects of variations in tooth morphology and position on eruption. Dent Pract Dent Rec. 1971;22:95-108.

13. Witsenburg B, Boering $G$. Eruption of impacted permanent upper incisors after removal of supernumerary teeth. Int J Oral Surg. $1981 ; 10: 423-431$.

14. Nagaraj K, Upadhyay M, Yadav S. Impacted maxillary central incisor, canine, and second molar with 2 supernumerary teeth and an odontoma. Am J Orthod Dentofac Orthop. 2009; 135:390-399.

15. Raupp S, Kramer PF, de Oliveira HW, da Rosa FM, Faraco IM Jr. Application of computed tomography for supernumerary teeth location in pediatric dentistry. J Clin Pediatr Dent. 2008;32:273-276.

16. Burden DJ, Mullally BH, Robinson SN. Palatally ectopic canines: Closed eruption versus open eruption. Am J Orthod Dentofac Orthop. 1999;1 15:634-639.

17. Kokich VG. Surgical and orthodontic management of impacted maxillary canines. Am J Orthod Dentofacial Orthop. 2004;126:278283.

18. Poyton GH, Morgan GH, Crouch SA. Recurring supernumerary mandibular premolars. Report of a case of postmature development. Oral Surg Oral Med Oral Pathol. 1960;1 3:964-966.

19. De Clerk Hugo, Cevidanes L, Baccetti T. Dentofacial effects of bone-anchored maxillary protraction : A controlled study of consecutively treated Class III patients. Am J Orthod Dentofacial Orthop. 2010;138:577-581.

20. Jackson DW. Interceptive Orthodontics: Early Treatment in Orthodontics. Journal of the American Orthodontic Society. 2009;9:12-15.

21. Batra P, Duggal R, Parkash H. Non-syndromic multiple supernumerary teeth transmitted as an autosomal dominant trait. J Oral Pathol Med. 2005;34:621-625. 